\title{
The tip-of-the-tongue phenomenon: Search strategy and resolution during word finding difficulties
}

Nina Jeanette Hofferberth

Department of German Linguistics, University of Wuppertal, Germany

https://doi.org/10.36505/ExLing-2011/04/0020/000189

\begin{abstract}
A tip-of-the-tongue (TOT) experience refers to the state in which a speaker is temporally unable to retrieve a word from memory, while being sure that he knows the word. The recovered partial information can consist of competing items that resemble the target word phonologically or semantically and could give rise to competition or conflict during attempts to resolve the TOT. A question that has been discussed recently is whether phonologically similar words block or facilitate lexical retrieval. A study at Frankfurt University (cf. Hofferberth 2008) found that participants preferred searching for their intended word by a semantic search strategy instead of a phonological one.
\end{abstract}

Key words: speech production, lexical access, word finding failures, blocking, incomplete activation

\section{The tip-of-the-tongue phenomenon}

The tip-of-the-tongue (TOT) state has been described as one ,in which one cannot quite recall a familiar word but can recall words of similar form and meaning“" (Brown \& McNeill 1966: 325). In a TOT state, speakers have a strong feeling of knowing the word, have access to its meaning and (partial) access to its syntactic properties. Speakers cannot retrieve the complete phonological form but are often able to retrieve the first letter, the number of syllables, stress pattern, other letters/phonemes in the word as well as words with similar sound and similar meaning.

Successful lexical retrieval in a TOT state can be imminent (within minutes) or delayed (after some hours or days) and may occur spontaneously (pop-ups). The resolution may be through external search strategies (such as looking up the word in a dictionary or by asking someone) or through internal strategies (such as searching in the alphabet or generating similar words) (cf. Brown 1991).

TOT states may represent the momentary unavailability of an otherwise accessible word (blocking hypothesis) or the weak activation of an otherwise inaccessible word (incomplete activation hypothesis) (cf. Meyer \& Bock 1992).

The recovered partial information could give rise to competition during attempts to resolve the TOT. For example, given the cue Aida Composer, subjects may recover the initial letter $V$, the final letter $I$ and know that it is a short Italian name and could then recall Vivaldi instead of Verdi (cf. Maril et al. 2001: 658).

ExLing 2011: Proceedings of 4th Tutorial and Research Workshop on Experimental Linguistics, 25-27 May, Paris, France 


\section{Experimental methodology}

A multiple single case study with German students at Frankfurt University examined the search strategy to resolve the TOT state (cf. Hofferberth 2008).

\section{Participants}

The participants in this experiment were five undergraduates at Frankfurt University who volunteered their services. All subjects were native speakers of German.

\section{Materials}

Participants attempted to retrieve 25 rare German target words (18 nouns, 2 verbs, 5 adjectives) from definition, such as "an instrument used for the application of capital punishment by decapitation" for guillotine. This study is a replication of the classic questionnaire study by Brown \& McNeill (1966). If the target word was known or unknown, the subject could write down the answer on the response sheet or leave it open. If the participant experienced a TOT state, he responded to a series of questions about initial, final and further letter(s) of the word, number of syllables, words with similar sound, words with similar meaning, word class, and any other data that came to mind (e.g., gender of a noun).

\section{Procedure}

All participants were tested together in one room. At the beginning of the experiment, each participant received a response sheet. The experimenter explained the tip-of-the-tongue phenomenon and read aloud the printed instructions. Then, the experimenter read the first definition aloud, and participants were allowed $90 \mathrm{sec}$ to fill out the response sheet. At the end of this period, the next definition was presented. This procedure was repeated for each of the 25 words in the list.

\section{Response coding}

The evaluation of positive TOTs (word recalled is correct) and unresolved TOTs (no word recalled) can be seen in figure 1. Negative TOTs (word recalled is incorrect) remain unconsidered because the information given by the participants refers to a non-target word.

\section{Results}

In $46.4 \%$ of the cases, the participants could immediately name the word; in $10.4 \%$ they did not know the word; and in $43.2 \%$ they experienced a TOT state $(14.1 \%$ positive TOTs, $11.1 \%$ negative TOTs and $64.8 \%$ unresolved TOTs). Participants preferred searching for their intended word by a semantic search strategy, i.e. via associations, context, co-hyponyms, such as Marie Antoinette, France, bastille, axe to retrieve guillotine. The results showed that participants used only marginally the phonological search 
strategy (searching in the alphabet $G . .$. or creating words with similar sound, such as gelatine to retrieve guillotine).

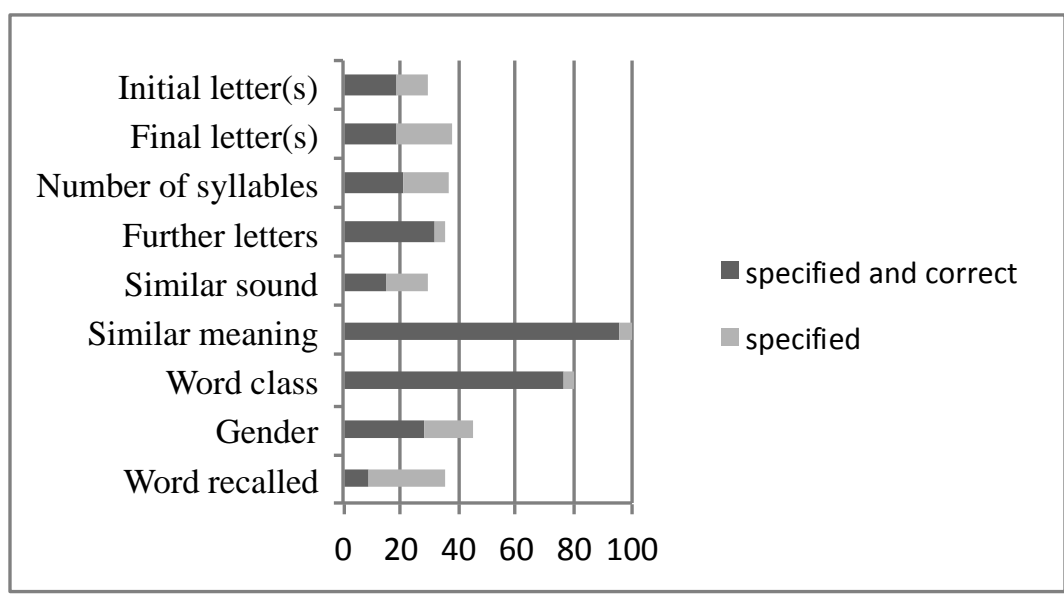

Figure 1. Evaluation of positive TOTs (word recalled is correct) and unresolved TOTs (no word recalled). Main result: All participants experiencing a TOT responded to the category 'words with similar meaning' $(100 \%), 98 \%$ of the information given was correct. The preference for resolving a TOT was a semantic search strategy and not a phonological one.

\section{Discussion}

The results show that participants used mostly the semantic search strategy by browsing semantic networks (specified in 100\%, correct in 98\%) and only hardly via phonologically similar words (specified in $29.2 \%, 50 \%$ correct). The preference for the semantic search strategy is more consistent with the blocking hypothesis and could be interpreted in a way that words with similar sound are usually not used to resolve the TOT state because they might block the target.

\section{General discussion and suggestions for future research}

TOTs are an important source of information concerning the nature of the processes and architecture of the speech production system. Models of lexical retrieval largely agree on the general distinction between semanticsyntactic (lemma) and word form retrieval processes (lexeme). They differ, however, in the precise architecture of the system. Currently under debate is the relationship between semantic, syntactic, and morpho-phonological units as well as the time-course of their activation.

Experiments by Jones (1989; see also Jones \& Langford 1987) and Maylor (1990) found that TOT states were more frequent when target and cue were phonologically related than when they were unrelated or only related in 
meaning. These results are more in line with the incomplete activation hypothesis. Meyer \& Bock (1992), on the other hand, showed that sound cues were more effective retrieval aids than meaning cues. These results are more consistent with the incomplete activation hypothesis.

The phonological cues used by Meyer \& Bock (1992) had the same initial sound and letter, the same number of syllables, and the same stress pattern as the targets but were unrelated to them in meaning (e.g., phonological cue ignorant for the target incubate). These phonological cues might not block lexical retrieval because they are less related to the target than words with similar rhyme (e.g., the phonological cue gelatine would probably block the target guillotine). In order to compare different phonological cues (such as the first syllable and the end rhyme) with semantic cues (such as associations and co-hyponyms), reaction time experiments with students at Dusseldorf University will commence in summer in order to examine the preference of the semantic search strategy any further.

\section{References}

Brown, A.S. 1991. A review of the tip-of-the tongue experience. Psychological Bulletin 109, 204-223.

Brown, R., McNeill, D. 1966. The 'tip of the tongue' phenomenon. Journal of Verbal Learning and Verbal Behaviour 5, 325-337

Hofferberth, N.J. 2008. Das Tip-of-the-Tongue-Phänomen. Eine multiple Einzelfallstudie. Frankfurt University: Unpublished master's thesis.

Jones, G.V. 1989. Back to Woodworth: Role of interlopers in the tip-of-the-tongue phenomenon. Memory \& Cognition 17, 69-76.

Jones, G.V., Langford, S. 1987. Phonological blocking in the tip of the tongue state. Cognition 26, 115-122.

Maril, A., Wagner, A.D., Schacter, D.L. 2001. On the tip of the tongue: An eventrelated fMRI study of semantic retrieval failure and cognitive conflict. Neuron 31, 653-660.

Maylor, E.A. 1990. Age, blocking and the tip-of-the-tongue state. British Journal of Psychology 81, 123-134.

Meyer, A.S., Bock, K. 1992. The tip-of-the-tongue phenomenon: Blocking or partial activation? Memory \& Cognition 20, 715-726. 